\title{
Ensayos de resistencia de pórticos de concreto a escala, reforzados con CFRP en los nudos
}

\section{Test concrete frames scale reinforced with CFRP in knots}

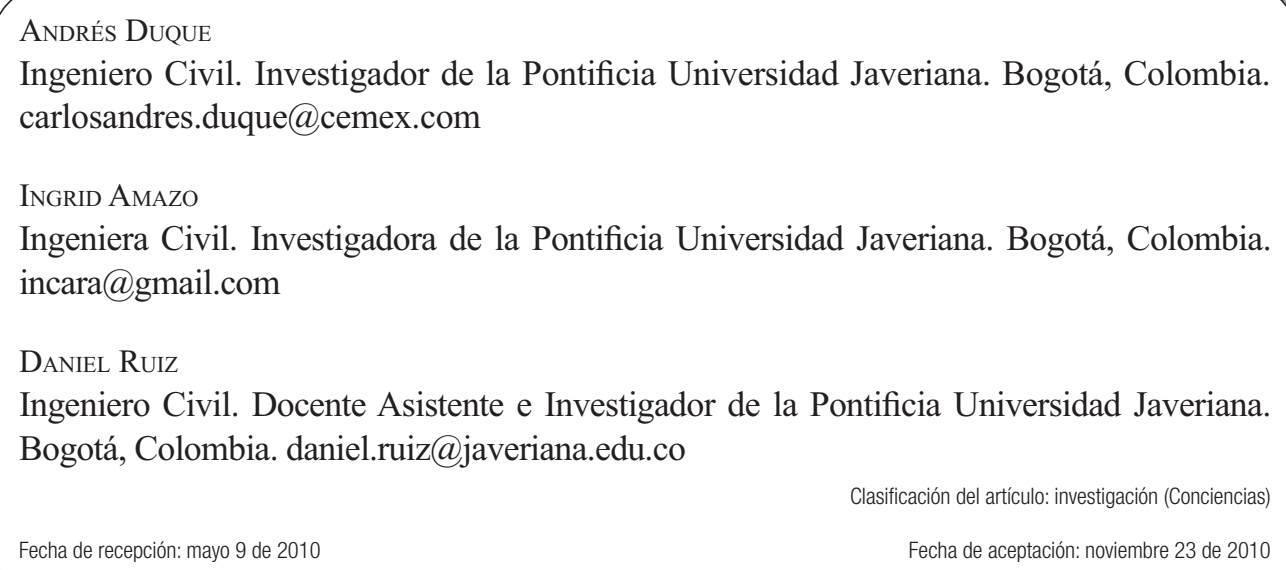

Palabras clave: Ensayos de laboratorio, Polimeros reforzados con fibra de carbono (CFRP), Refuerzo sismico exterior.

Key words: Laboratory test, Carbon fiber reinforced polymers (CFRP), External seismic reinforcement.

\section{Resumen}

Se presentan los resultados de un estudio experimental de cuatro pórticos de concreto a escala (1:2) sometidos a carga monotónica. Éstos fueron diseñados y construidos sin zonas de confinamiento en los nudos y considerando únicamente la carga gravitacional. Dos de los cuatro marcos de concreto fueron fortalecidos en los nudos mediante un confinamiento con Polímeros Reforzados con Fibra de Carbono (CFRP). La instrumentación de los ensayos consistió en una celda de carga, deformímetros análogos y strain gages en las barras de acero de refuerzo y en las fibras de carbono. De acuerdo con los resultados experimentales, el refuerzo con fibras duplica la resistencia y la rigidez de los pórticos e incrementa su desplazamiento máximo sin pérdida de resistencia en un $60 \%$. Así mismo, el confinamiento de los nudos con CFRP disminuye notablemente las fisuras y grietas de los elementos estructurales.

\begin{abstract}
The results of an experimental study of four scale concrete frames (1:2) subjected to monotonic load are presented. The four frames were designed and constructed without confinement zones in the
\end{abstract}




\section{con-ciencias}

joints and considering only the gravitational load. Two of the four concrete frames of concrete were reinforced in the joints with a confinement with polymers reinforced with carbon fiber (CFRP). The instrumentation of the tests consisted of a load cell, analogous deformimeters and strain gages in the reinforcement steel bars and in the carbon fibers. According to the experimental results, the reinforcement with fibers duplicates the resistance and the rigidity of the frames and increases its total displacement without loss of resistance in $60 \%$. Also the confinement of the joints with CFRP diminishes remarkably the fissures and the cracks of the structural elements.

\section{Introducción}

El trauma causado por el colapso parcial o total de las estructuras hechas por el hombre es la causa más común de muerte y lesión en la mayoría de los terremotos. Según Gallego y Yamín (2002) cerca del $75 \%$ de las muertes atribuidas a terremotos en Colombia en el siglo XX fueron causadas por el colapso de edificaciones que no tuvieron un adecuado diseño sismo resistente, construidas con materiales inapropiados o simplemente arrasados por la capacidad destructiva de un sismo de grandes proporciones. Es muy común encontrar en Colombia hospitales, entidades públicas, puentes y colegios (con estructura en pórticos de concreto) que fueron construidos antes de la existencia de códigos de construcción sismo resistentes y que no tuvieron en cuenta criterios de detallamiento sísmico. Estas edificaciones en concreto deben ser reforzadas y reparadas para poder garantizar la seguridad de los usuarios, pero esto debe hacerse mediante técnicas que, en lo posible, no impliquen el cierre de las instituciones.

Ante este panorama una de las alternativas, que está tomando la delantera en la rehabilitación de estructuras, son los Polímeros Reforzados con Fibras de Carbono (CFRP), los cuales tienen una alta resistencia a la tensión, son de bajo peso, no se corroen y permiten el normal funcionamiento de una estructura durante la fase de reparación, gracias a que las resinas adquieren alta resistencia a edades tempranas. Adicionalmente, su proceso de instalación es sencillo y no requiere de mano de obra demasiado especializada.

\section{Refuerzo de estructuras de concreto con CFRP}

Los materiales compuestos FRP (Fiber Reinforced Polymer) son fibras de gran resistencia a la tensión, formadas por tejidos de fibra de carbono (CFRP), vidrio (GFRP) o aramida. Estos compuestos se adhieren con una resina epóxica a los elementos estructurales como columnas, vigas, losas, etcétera, protegiéndolos del medio ambiente y generando mejoras de resistencia y de ductilidad. La fibra aporta rigidez y resistencia, y la matriz configura geométricamente el material compuesto. Según Gómez y Sobrino [1] la matriz es poco resistente en comparación con las fibras y tiene la misión principal de transmitir los esfuerzos de unas fibras a otras, y entre ellas y la superficie adyacente.

La resistencia de un material compuesto depende del porcentaje de las fibras en volumen, de las propiedades mecánicas de la matriz, de la adherencia entre fibra y matriz y, sobre todo, de la dirección en la que se ubican las fibras. Si éstas se encuentran orientadas en una única dirección la máxima resistencia y el módulo de deformación mayor se encontrará en dicha dirección. Una disposición de las fibras en forma de "tejido" (refuerzo en dos direcciones) genera diferentes resistencias según el ángulo formado por las fibras. 


\section{con-ciencias}

A nivel mundial se han realizado diversas investigaciones y aplicaciones para mejorar el comportamiento de estructuras de concreto a cortante y flexión, como se muestra en Yost, Goodspeed y Schmeckpeper [2]. En Europa el líder en desarrollo y aplicación de FRP es Suiza y el primer reforzamiento de un puente en el mundo utilizando platinas de carbono se hizo en 1991 en el puente Ibach en Lucerna [1]. Por otro lado, en los Estados Unidos, en 1995, el 42\% de los puentes de autopistas necesitaban ser rehabilitados y por esto al programa de rehabilitación de puentes, que empezó en 1995, se le han destinado dos billones de dólares. El 40\% de este dinero ha sido invertido en aplicaciones con FRP, en las que se encamisaron columnas con CFRP, como se muestra en [3]. Según SIKA [4] la primera aplicación con FRP en Latinoamérica se hizo en Colombia en 1996, en el puente Cocorná en la vía Bogotá-Medellín, utilizando platinas de carbono. Después de esta experiencia han seguido otras como el refuerzo del puente de la calle 13 por la carrera 30 con platinas de carbono, el de la planta de agua potable de la planta Vitelma, el sísmico de escuelas distritales (como el colegio Manuelita Sáenz), el teatro Los Fundadores (en Manizales), edificios de muros estructurales en apartamentos en Barraquilla, el Centro Comercial Multicentro (Ibagué), el edificio del Centro Administrativo Distrital, un puente en Suesca (Cundinamarca), entre otros.

Así mismo, diversas investigaciones han demostrado que el confinamiento del concreto mediante FRP aumenta de manera importante tanto la ductilidad como la resistencia de probetas sometidas a esfuerzos de compresión, como se puede observar en Campione y Miraglia (2006), [5], [6], [7], [8], [9] y [10], entre otros.

En la Figura 1, tomada [6] se muestran curvas esfuerzo vs. deformación unitaria de especímenes de concreto con forma rectangular y circular, sometidos a la compresión con y sin confinamiento en FRP. Obsérvese que la probeta confinada con FRP tiene, en general, una resistencia superior a la de la probeta sin ningún confinamiento, así como una capacidad de deformación mayor sin pérdidas de resistencia. Las probetas reforzadas con las fibras fallaron por las fibras o por el desprendimiento de las mismas.

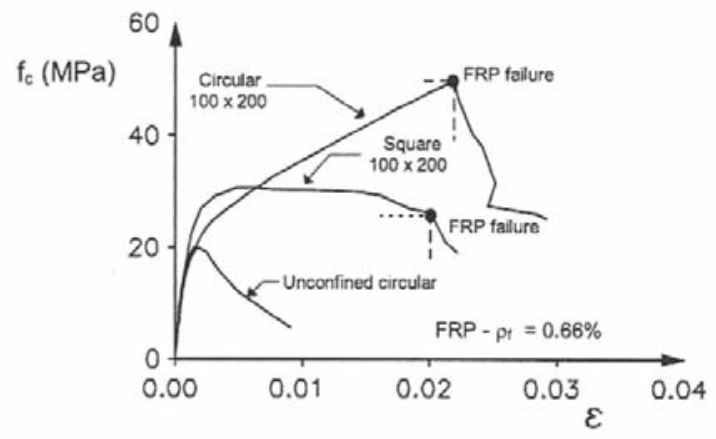

Figura 1. Curvas esfuerzo-deformación para concreto con y sin confinamiento con FRP y con diferentes formas [6].

Por otro lado, existen diversos estudios en la literatura que reportan resultados experimentales sobre modelos a escala natural y reducida de elementos de concreto reforzados con FRP sometidos a flexión y a flexocompresión. Ejemplo de estas pruebas de laboratorio se pueden encontrar en [11],[12], [13], [11] y [14]. La gran mayoría de las investigaciones experimentales se han realizado sobre elementos tipo columna en voladizo (Figura 2), sometidas a cargas cíclicas y con refuerzo en FRP tanto en el nudo que soporta el momento máximo como a lo largo de toda la columna.

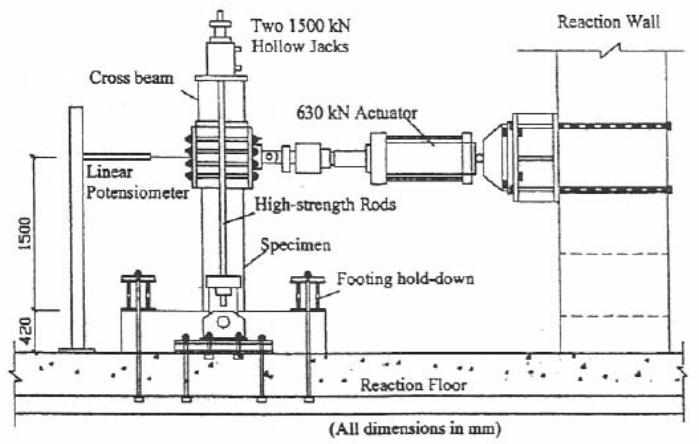

Figura 2. Montaje de ensayo cíclico típico de columnas reforzadas con CFRP y GFRP. Tomado de [11]. 


\section{con-ciencias}

También en la literatura técnica se encuentran estudios del refuerzo de nudos viga-columna ante cargas seudodinámicas, como se muestra en [15] [16] y [17]. En estos ensayos se reconstruyeron nudos típicos de pórticos y se rehabilitaron con diferentes esquemas de aplicación de las FRP. Posteriormente, estos nudos se sometieron a cargas cíclicas estableciendo los ciclos de histéresis. En particular, se destacan los resultados de [15]. En este estudio se reconstruyeron nudos columna-viga-columna, como se muestra en la Figura 3.

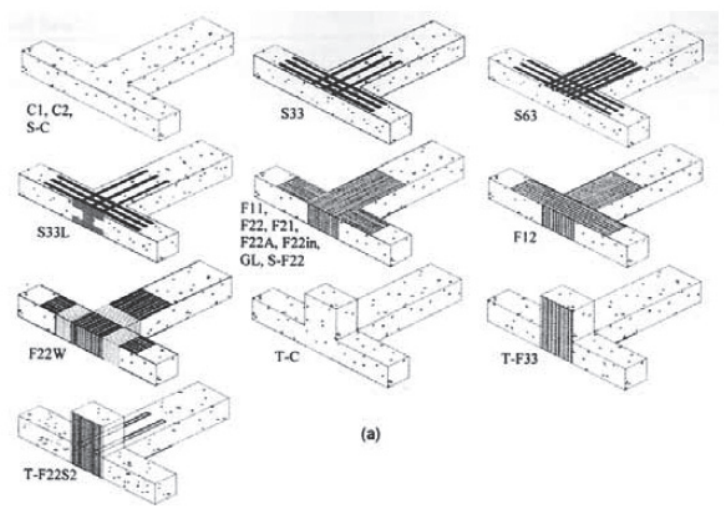

Figura 3. Ejemplo de uniones viga-columna reforzados con CFRP, ensayados por [15].

Como podrá observarse, no abunda en los reportes de investigación pruebas experimentales sobre pórticos de concreto completos (al menos dos columnas y una viga) reforzados con FRP en los nudos. Estas pruebas experimentales son necesarias para verificar el comportamiento de todo el sistema estructural (nudos, vigas y columnas simultáneamente). Es por esto que con el apoyo de una empresa del sector productivo se planteó un proyecto de investigación con el fin de evaluar experimentalmente la rehabilitación sísmica de estructuras aporticadas de concreto, mediante el confinamiento de los nudos con CFRP. Para ello se diseñó un experimento de carga monotónica hasta la falla, como el mostrado en la Figura 4.

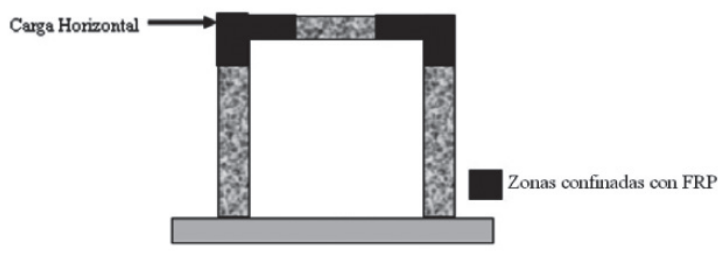

Figura 4. Esquema de los ensayos experimentales desarrollados.

\section{Metodología}

\subsection{Construcción de los pórticos a escala}

Con el fin de evaluar el comportamiento del confinamiento de nudos de pórticos de concreto reforzado mediante fibras de carbono, se construyeron cuatro pórticos a escala 1:2 de una luz y un piso de altura. En estos ensayos experimentales no se buscó extrapolar los resultados a pórticos a escala natural sino únicamente realizar análisis comparativos entre el comportamiento mecánico de los pórticos reforzados con CFRP con sus equivalentes sin refuerzo.

Los pórticos a escala natural fueron diseñados sin ningún requisito de confinamiento o ductilidad para simular el comportamiento de sistemas estructurales en concreto construidos antes de la vigencia de la norma sismo resistente del año 1984 [18]. Tanto la luz de la viga como la altura de las columnas en los modelos a escala fue de $1.35 \mathrm{~m}$. La sección transversal de los tres elementos estructurales a escala fue cuadrada, de $15 \mathrm{~cm}$ de lado y cada elemento tenía como refuerzo principal cuatro barras de acero de $9.5 \mathrm{~mm}$ de diámetro (3/8), con una altura efectiva del refuerzo de $13.5 \mathrm{~cm}$. Se debe mencionar que se mantuvo la cuantía de vigas y columnas entre el pórtico a escala real y sus equivalentes a escala 1:2. Así mismo, los estribos en los modelos estaban conformados por barras lisas de $6.35 \mathrm{~mm}$ de diámetro $(1 / 4)$, espaciadas cada $8 \mathrm{~cm}$. El acero de refuerzo de las barras de refuerzo principal tuvo un esfuerzo de fluencia de $420 \mathrm{MPa}$ y el de los estribos de 240 $\mathrm{MPa}$, lo cual fue corroborado a través de pruebas a tensión realizadas en el Laboratorio de Pruebas 


\section{con-ciencias}

y Ensayos (acreditado ante la Superintendencia de Industria y Comercio) de la Universidad Javeriana. En la Figura 5a se muestra el detalle de la canasta refuerzo antes de fundir el concreto. Es importante mencionar que los pórticos se diseñaron de tal manera que fuesen articulados en la base; para ello se creó una rótula física al cruzar en la mitad de la altura de la columna, el refuerzo longitudinal como se muestra en la Figura 5b. De esta manera, se garantizó una concentración de los daños por flexión en los nudos viga-columna y no en las zonas de soporte. Los elementos tipo rótula fueron posteriormente unidos con el resto del refuerzo, garantizando las longitudes de traslapo estipuladas en [19]. Como se verá más adelante, este detalle funcionó de la manera esperada.

a)
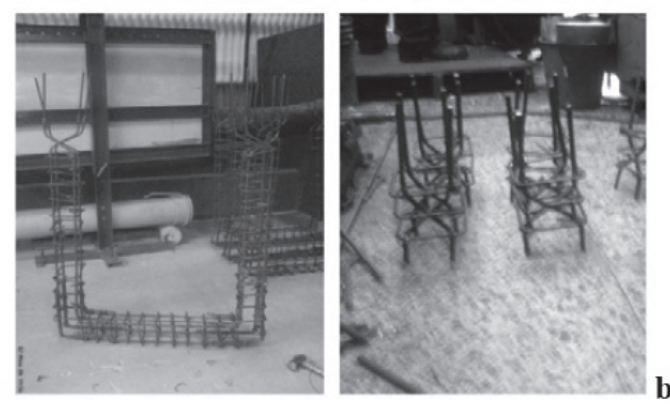

Figura 5. a). Detalle de la canasta de refuerzo b). Detalle de rótula física de la cimentación.

Con el fin de establecer deformaciones unitarias en el refuerzo principal de acero se instalaron strain gages (galgas extensométricas) uniaxiales en el refuerzo, ubicado en la zona de los nudos. Este proceso se realizó antes de fundir el concreto y, de esta manera, se registraron las deformaciones del acero durante el proceso de carga. En la Figura 6 se muestra el detalle de los strain gages usados y se muestran las galgas ya instaladas.

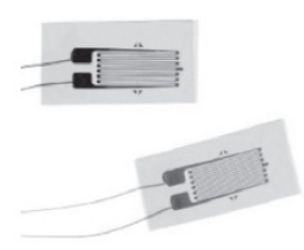

a)

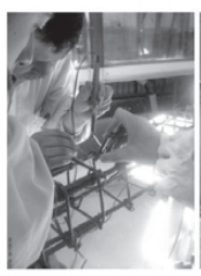

b)

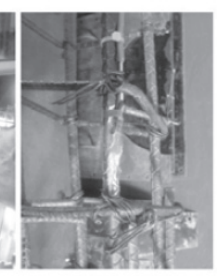

c)
Figura 6. a) Ejemplo de galgas extensométricas b) Proceso de instalación de los strain gages. c)Strain gages instalados en el refuerzo de acero.

Con el refuerzo y la instrumentación instalada se procedió a fundir el concreto que fue diseñado con una resistencia de $21 \mathrm{MPa}$ a los 28 días. En la figura 7a se muestra la formaleta de dos de los pórticos y, posteriormente, en la figura $7 \mathrm{~b}$ se presenta el pórtico ya fundido. Se tomaron cuatro cilindros de concreto testigo para determinar su resistencia a la compresión.

Para la aplicación de las cargas, el Laboratorio de Pruebas y Ensayos cuenta con un sistema de gatos hidráulicos unido a una máquina universal de ensayos. La carga aplicada por los gatos reacciona contra un marco de acero (Figura 8a). En las Figuras $8 \mathrm{~b}$ y $8 \mathrm{c}$ se muestra un esquema de los pórticos de concreto instalados en el marco de reacción. Obsérvese que para el montaje es necesario girar 90 grados los pórticos de concreto aunque se debe aclarar que el peso de los pórticos se incluyó dentro de la precarga de los pórticos. Así mismo, debe recordarse que los nudos de la zona inferior del pórtico no transmiten momento al marco de carga sino únicamente cortante y carga axial. 


\section{con-ciencias}

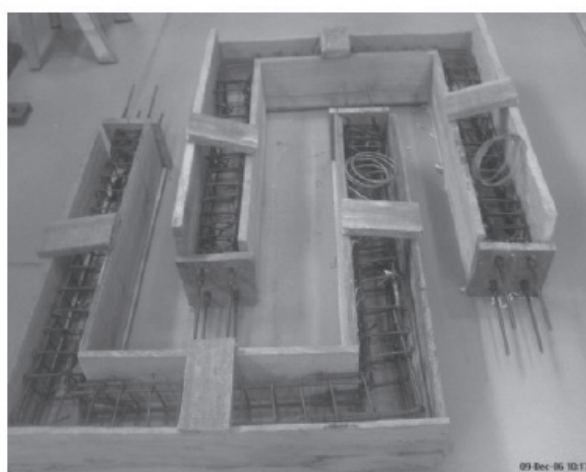

a)

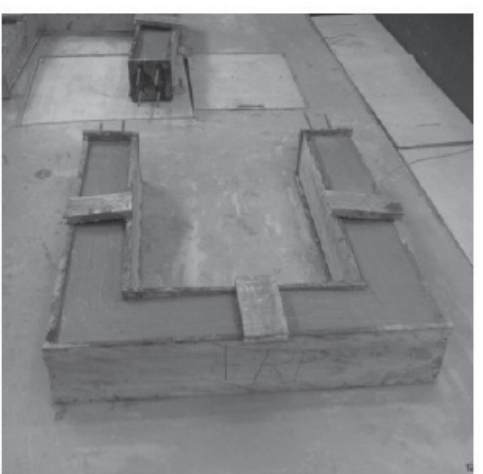

b)

Figura 7. a) Formaleta con la canasta de refuerzo y la instrumentación instalada.

b). Formaleta con el concreto fundido.

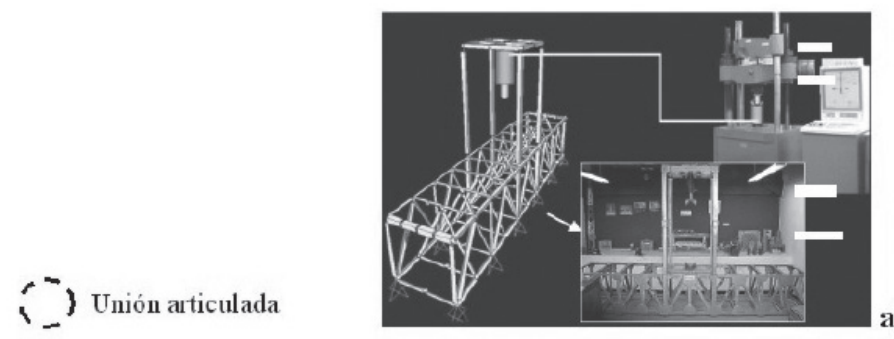

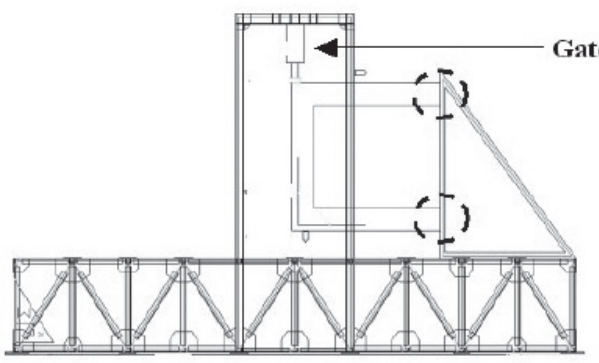

b)

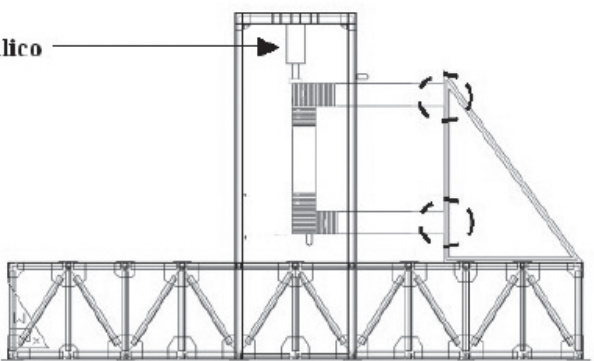

c)

Figura 8. a). Esquema del sistema de aplicación de carga.

b). Esquema de aplicación de carga sobre pórticos sin refuerzo.

c). Esquema de aplicación de carga sobre pórticos reforzados con CFRP.

\subsection{Refuerzo con fibras de carbono}

Para el reforzamiento sísmico de los nudos se utilizaron fibras de carbono unidireccionales con las características mecánicas que se presentan en la Tabla 1 (suministradas por el fabricante). Las fibras de carbono de los pórticos reforzados fueron instaladas 48 horas antes de ejecutar las pruebas en el marco de reacción.

Para la instalación de las fibras, se contó con la asesoría de la empresa fabricante de las fibras, si- 


\section{con-ciencias}

guiendo todos los procesos y las recomendaciones técnicas del caso. Para ello se pulió la superficie de los nudos y los vértices fueron redondeados (Figura 9a). Previamente, las fibras de carbono fueron cortadas a la medida (Figura 9b) para posteriormente instalarlas envolviendo el nudo. En esta etapa fue de suma importancia la resina epóxica, suministrada por el mismo proveedor de las fibras.

Tabla 1. Propiedades de las fibras de refuerzo.

\begin{tabular}{|c|c|}
\hline Peso & $3 \mathrm{~N} / \mathrm{m} 2$ \\
\hline Ancho del rollo & $600 \mathrm{~mm}$ \\
\hline Longitud del rollo & $50 \mathrm{~m}$ \\
\hline Espesor de diseño del tejido & $0.17 \mathrm{~mm}$ \\
\hline Resistencia a la tensión del tejido & $3900 \mathrm{~N} / \mathrm{mm} 2$ \\
\hline Módulo de elasticidad del tejido & $230.000 \mathrm{~N} / \mathrm{mm} 2$ \\
\hline Elongación de rotura & $1.5 \%$ \\
\hline
\end{tabular}

Para la instalación se tuvo cuidado de eliminar cualquier bolsa de aire o irregularidad utilizando rodillos de caucho (Figura 9c), impregnando en su totalidad la resina a las fibras y dejando una pequeña zona de transición entre la zona reforzada y sin refuerzo. Se instalaron los CFRP en los nudos, en las direcciones ilustradas en las Figuras 9d y 9e. Aparte del nudo como tal, la longitud reforzada con CFRP de los elementos estructurales fue de $20 \mathrm{~cm}$ desde la cara de la viga y de la columna. Es importante mencionar que el confinamiento que se dio a los nudos es el mejor posible, ya que fueron recubiertos en su totalidad. Es probable que éste no pueda aplicarse con la misma facilidad en pórticos de concreto reales, dejando así la investigación de estos esquemas de refuerzo para trabajos futuros. Por último, se instalaron en los nudos strain gages biaxiales (como se muestra en la Figura 9e) con uno de sus ejes en dirección de las fibras y el otro a 45 grados.

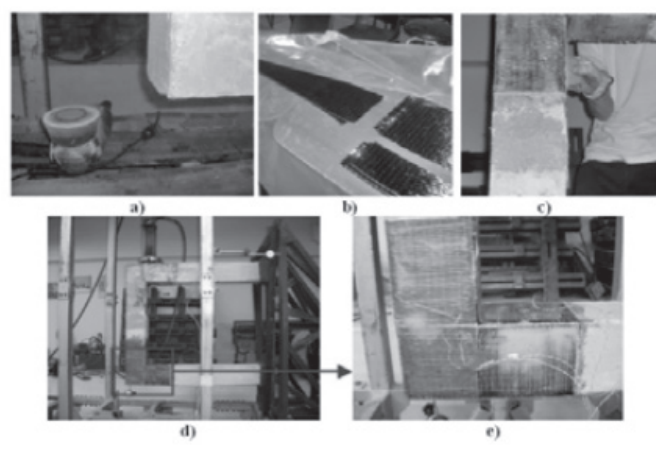

Figura 9. a). Superficies pulidas y esquinas redondeadas. b). Fibras de carbono cortadas a la medida.

c). Instalación de las fibras y del epóxico. 9d y 9e. Detalle de fibras instaladas en los pórticos.

\section{Resultados}

Antes de efectuar las pruebas de carga paralela al plano del pórtico se realizó un control de la resistencia del concreto mediante la compresión de cilindros de $76 \mathrm{~mm}$ de diámetro (3), ensayados a los 28 días al igual que los pórticos. En la Tabla 2 se muestran los esfuerzos resistentes que son superiores al diseño $(21 \mathrm{MPa})$.

A los 28 días de construidos fueron ensayados los pórticos ante carga monotónica creciente paralela al plano principal. Una vez instalado cada pórtico en el marco de reacción (Figura 10a) se procedió a instrumentarlo mediante deformímetros análogos calibrados (precisión de $0.01 \mathrm{~mm}$ ), como se muestra en las Figuras 10b y 10c. Éstos registraban la deriva del pórtico así como los movimientos relativos de los apoyos. Mediante un sistema de adquisición de datos (Figura 10d) se tomaron los registros de las deformaciones unitarias en los strain gages instalados en las barras de acero y en las fibras de refuerzo. El sistema de gatos hidráulicos, que está unido a la máquina universal de ensayos, registra automáticamente la carga aplicada con una celda de carga con una precisión de $+/-20 \mathrm{~N}$. 


\section{con-ciencias}

Tabla 2. Ensayos de compresión de cilindros testigo.

\begin{tabular}{|c|c|c|}
\hline N $^{\circ}$ MUESTRA & TIPO DE FALLA & RESISTENCIA (MPa) \\
\hline Cilindro 1 & Corte & 24 \\
\hline Cilindro 2 & Corte & 29 \\
\hline Cilindro 3 & Corte y cono & 31 \\
\hline Cilindro 4 & Corte y cono & 26 \\
\hline Promedio & & 27 \\
\hline
\end{tabular}

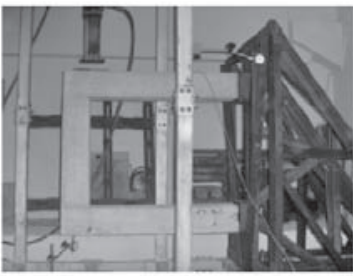

a)

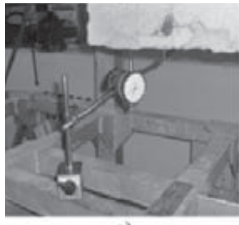

c)

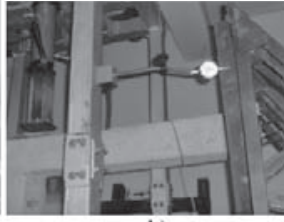

b)

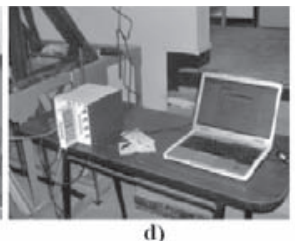

d)
Figura 10. a). Pórtico instalado en el marco de reacción. b) y c). Deformímetros análogos para medición de desplazamientos. d). Sistema de adquisición de datos para las deformaciones unitarias.

Se procedió a realizar la prueba mediante control de desplazamiento (control de deriva del pórtico), registrando en cada incremento la fuerza aplicada, el desplazamiento de los deformímetros y las deformaciones unitarias en los strain gages. Simultáneamente, se tomó un registro fotográfico detallado de la evolución de las fisuras y posteriores grietas de los elementos estructurales. En la Figura 11 se muestran detalles de la prueba efectuada sobre uno de los dos pórticos sin fibras de carbono de refuerzo. En la Figura 11a se muestra el pórtico al inicio de la prueba, en las Figuras 11 b y $11 \mathrm{c}$ se da a conocer la evolución de las fisuras en los nudos, las cuales culminan con agrietamientos excesivos que pueden observarse en la Figura 11d. Finalmente, en la Figura 11e se puede apreciar el estado de falla del pórtico, el cual se manifestó con una pérdida excesiva de resistencia. Es importante anotar que los apoyos de segundo grado se comportaron de acuerdo con lo establecido en el diseño experimental y no se detectaron grietas en dicha zona en ninguna de las pruebas realizadas.

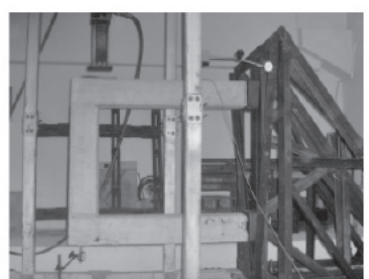

a)

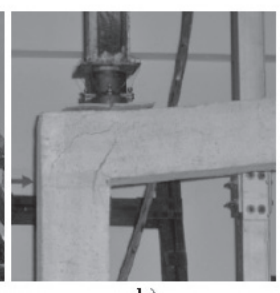

b)

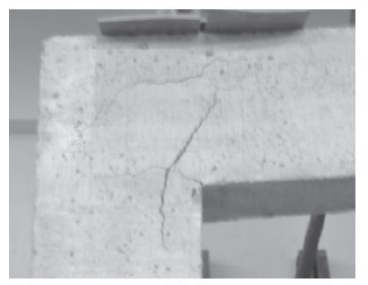

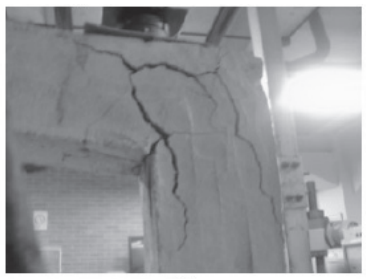

d)

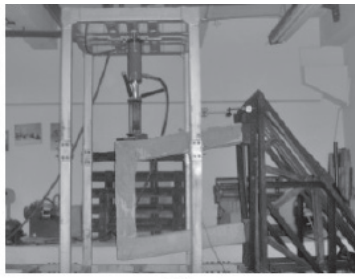

e)
Figura 11. a). Montaje del pórtico antes del ensayo. b) y c). Fisuras en los nudos.

d). Agrietamiento del nudo en el momento de la falla. e). Estado del pórtico al finalizar la prueba.

Por otro lado, en las fotografías de la Figura 12 se muestran detalles de la prueba efectuada sobre uno de los pórticos reforzados con fibras de carbono en los nudos. El montaje se presenta en la Figura 12a. El procedimiento de ensayo y el control de la prueba mediante desplazamiento fueron similares a los seguidos con los pórticos sin rehabilitación. A la luz de los resultados, en los pórticos reforzados disminuyó sustancialmente la fisuración en comparación con los pórticos sin fibras de carbono. La falla se generó 


\section{con-ciencias}

en las inmediaciones de la zona donde se suspendía el refuerzo con CFRP, generándose una grieta por flexión en las columnas que atravesaba el elemento estructural de lado a lado (12b y 12c). No obstante, lo más destacable de las imágenes de la Figura 12 es la ausencia de fisuración en los nudos del pórtico, lo cual demuestra que las fibras confinaron adecuadamente las uniones viga-columna. Esto se corroboró al desprender (con bastante trabajo) los CFRP de uno de los nudos después de efectuadas las pruebas.
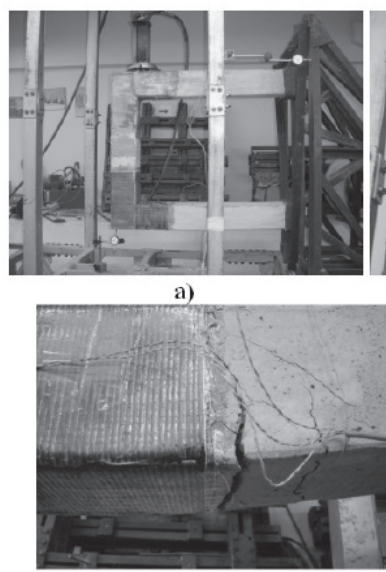

c)

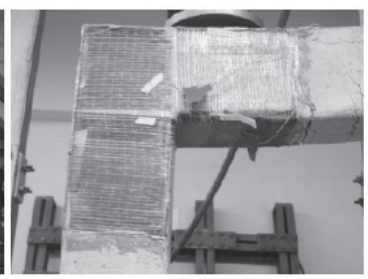

b)

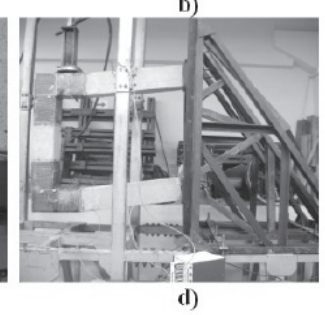

d)
Figura 12. a). Montaje del pórtico antes del ensayo. b) y c). Fisuras en las columnas.

d). Estado del pórtico al finalizar la prueba.

Por otra parte, en la Figura 13 se muestran los resultados de fuerza y de desplazamiento (deriva) de los pórticos ensayados. En ésta se observa que los pórticos reforzados con CFRP alcanzaron desplazamientos máximos 1.6 veces más grandes, sin pérdida de resistencia, que los desplazamientos de los pórticos sin refuerzo. Estos resultados son similares a los obtenidos por Xiao y Ma (19) y por Xiao, Wu y Martín (20). No obstante, debe observarse que las derivas alcanzadas son bastante grandes (llegan hasta el 12\%) debido, principalmente, a las condiciones de apoyo de segundo grado (no de empotramiento) de los pórticos. Esta condición de frontera genera grandes giros en los apoyos que se manifiestan en las grandes derivas. Por otro lado, en promedio, los pórticos reforzados con CFRP tienen una fuerza resistente que duplica (2.1 veces) la fuerza máxima aplicada a los pórticos sin refuerzo. Estos incrementos de resistencia son similares a los obtenidos por Antonopoulos y Triantafillou [15], en donde los nudos reforzados con CFRP resistieron 1.9 veces lo que sus similares sin refuerzo.

Otro aspecto importante que se observa en la Figura 13 es el aumento de la rigidez de los pórticos reforzados con CFRP, con respecto a los pórticos sin refuerzo. En promedio, la rigidez de los pórticos reforzados con fibras de carbono es 1.93 veces la rigidez de los pórticos de concreto sin refuerzo.

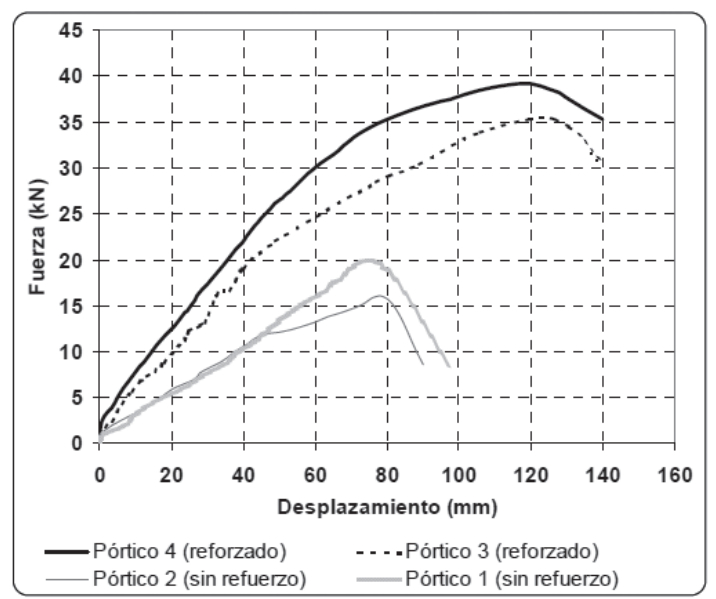

Figura 13. Curvas de fuerza vs. desplazamiento de los pórticos ensayados

Así mismo, las deformaciones unitarias máximas en los strain gages ubicados en los elementos de acero alcanzaron valores de $0.87 \%$ y $0.77 \%$ en los pórticos uno y dos (sin CFRP) respectivamente, que al compararlos con el $0.21 \%$ de esfuerzo de fluencia implica que las barras de acero salieron del rango elástico. Las deformaciones unitarias en el acero de refuerzo en los pórticos tres y cuatro (con CFRP) alcanzaron valores de $1.1 \%$ y $3.65 \%$ respectivamente, lo cual implica que estas barras también fluyeron, pero incursionando en deformaciones inelásticas más grandes. Esto es consistente con los resultados globales a nivel de fuerza y des- 


\section{con-ciencias}

plazamiento de los pórticos de la 13. Finalmente, las deformaciones unitarias en los FRP en dirección de las fibras llegaron hasta el $0.65 \%$, valor inferior al $1.5 \%$ de deformación de rotura, según los datos suministrados por el fabricante.

\section{Conclusiones}

De acuerdo con los resultados experimentales se plantean las siguientes conclusiones:

- La alternativa de refuerzo con CFRP es viable y fácil de implementar en estructuras de concreto debido a la limpieza del proceso, al bajo peso de las fibras y a la rapidez del proceso.

- Al confinar los nudos de pórticos de concreto (diseñados originalmente sin requisitos de ductilidad en los nudos) con CFRP, se disminuye notablemente el agrietamiento de los pórticos de concreto reforzado. Así mismo, el reforzamiento cambia el esquema de falla de los pórticos a grietas originadas por flexión en la zona en donde se interrumpe el refuerzo con CFRP.

- Los pórticos reforzados con CFRP alcanzaron desplazamientos máximos (sin pérdida de resistencia) que son $60 \%$ superiores a los desplazamientos de los pórticos sin refuerzo.

- En promedio, la rigidez de los pórticos reforzados con fibras de carbono es 1.93 veces la rigidez de los pórticos de concreto sin refuerzo.
- En promedio, la fuerza máxima resistente de los pórticos reforzados con CFRP es 2.1 veces la fuerza resistente de los pórticos sin refuerzo.

\section{Agradecimientos}

Los autores del artículo agradecen la colaboración brindada por la empresa SIKA y, en particular, los aportes y la asesoría del ingeniero Jorge Rendón. Así mismo, agradecen al Ingeniero las gestiones que realizó para la consecución de los CFRP que sirvieron para reforzar los nudos de los pórticos de concreto.

De igual manera, los autores agradecen la colaboración prestada por el personal técnico y profesional del Laboratorio de Pruebas y Ensayos del Departamento de Ingeniería Civil de la Pontificia Universidad Javeriana.

\section{Trabajos futuros}

- Se estudiarán experimentalmente otros esquemas de refuerzo de los nudos en pórticos a escala real y a escala reducida.

- Se realizarán ensayos seudodinámicos de pórticos a escala reforzados en los nudos con CFRP.

\section{Referencias bibliográficas}

[1] M. Gómez, J. Sobrino, “Criterios de diseño para el refuerzo de estructuras con materiales compuestos con fibra de carbono. Casos prácticos", Revista de la facultad de Ingeniería de la Universidad de los Andes, no. 18, pp. 85-99, 2002.
[2] J. Yost, C. Goodspeed, E. Schmeckpeper, "Flexural performance of concrete beams reinforced with FRP grids", Journal of Composites for Construction, vol. 5, no. 1, p. $18,2001$. 
[3] I. Gergely, C. Pantelides, R. Nuismer, L. Reaveley, "Bridge pier retrofit using fiberreinforced plastic composites", Journal of Composites for Construction, vol. 2, no. 4, pp. 165-174, 1998.

[4] SIKA, Sika Noticias: Proyectos de Reforzamiento en Colombia con Materiales Compuestos FRP Sika ${ }^{\circledR}$ Carbodur ${ }^{\circledR}, 2006$.

[5] R. Kumutha, R. Vaidyanathan, M. Palanichamy, "Behaviour of reinforced concrete rectangular columnas strengthened using GFRP", Cement \& Concrete Composites, no. 29, pp. 1-30, 2007.

[6] G. Campione, "Influence of FRP wrapping techniques on the compressive behavior of concrete prisms", Cement \& Concrete Composites, no. 28, pp.497-505, 2006.

[7] F. Braga, R. Gigliotti, M. Laterza, "Analytical Stress-Strain Relationship for Concrete Confined by Steel Stirrups and/or FRP Jackets", Journal Of Structural Engineering, vol. 132, no. 9, 2006.

[8] M. Karantzikis, C. Papanicolaou, C. Antonopoulos, T. Triantafillou, Journal of Composites for Construction, vol. 9, no. 6, 2005.

[9] N. Sáenz, C. Pantelides, "Strain-based confinement model for FRP-confined concrete", Journal of Sytructural Engineering, vol. 133, no. 6, pp. 825-833, 2007.

[10] S. Pessiki, K. Harries, J. Kestner, R. Sause, J. Ricles, "Axial Behavior of reinforced concrete columns confined with FRP jackets", Journal of Composites for Construction, vol. 5, no. 4, pp. 237-245, 2001.

[11] B. Shan, Y. Xiao, Y. Guo, "Residual performance of FRP-Retrofitted RC columns after being subjected to cyclic loading Damage",

\section{con-ciencias}

Journal of Composites for construction, vol. 10, no. 4, pp. 304-312, 2006.

[12] Shamin, Sheik y Yau, "Seismic Behavior of Concrete Columns Confined with Steel and Fiber-Reinforced Polymers", ACI Structural Journal, Title no. 99-S8, 2002.

[13] Y. Xiao, H. Wu, G. Martin, "Prefabricated composite jacketing of RC columns for enhaced shear strength", Journal of structural engineering, vol. 125, no. 3, pp. 255-264, 1999.

[14] Y. Xiao, R. Ma, "Seismic retrofit of RC circular columns using prefabricated composite jacketing", Journal of structural engineering, vol. 123, no. 10, pp. 1357-1364, 1997.

[15] C. Antonopoulos, T. Triantafillou, "Experimental investigation of FRP-strengthened RC beam-column joints", Journal of composites for construction, vol. 7, no. 1, pp. 39-49, 2003.

[16] J. Gergely, C. Pantelides, L. Reaveley, "Shear strengthening of RCT-Joints using CFRP composites", Journal of composites for construction, vol. 4, no. 2, pp. 56-64, 2000.

[17] A. Prota, A. Nanni, G. Manfredi, E. Cosenza, "Selective upgrade of underdesigned reinforced concrete beam-column joints using carbon fiber-reinforced polymers". ACI structural journal, vol. 101, no. 5, pp. 699-707, 2004.

[18] SCI (Sociedad Colombiana de Ingenieros), Código Colombiano de Construcciones Sismo-Resistentes, 1984.

[19] AIS (Asociación Colombiana de Ingeniería Sísmica), Normas Colombianas de Diseño y Construcción Sismo Resistente (NSR-98). Bogotá, Colombia, 1998. 